Draft version February 1, 2008

Preprint typeset using LATEX style emulateapj v. 04/03/99

\title{
PROTON CYCLOTRON FEATURES IN THERMAL SPECTRA OF ULTRA-MAGNETIZED NEUTRON STARS
}

\author{
S. Zane ${ }^{1}$, R. Turolla ${ }^{2}$, L. Stella ${ }^{3}$, A. Treves ${ }^{4}$ \\ Draft version February 1, 2008
}

\begin{abstract}
A great deal of interest has been recently raised in connection with the possibility that soft $\gamma$ ray repeaters (SGRs) and anomalous X-ray pulsars (AXPs) contain magnetars, young neutron stars endowed with magnetic fields $\gtrsim 10^{14} \mathrm{G}$. In this paper we calculate thermal spectra from ultra-magnetized neutron stars for values of the luminosity and magnetic field believed to be relevant to SGRs and AXPs. Emergent spectra are found to be very close to a blackbody at the star effective temperature and exhibit a distinctive absorption feature at the proton cyclotron energy $E_{c, p} \simeq 0.63\left(B / 10^{14} \mathrm{G}\right) \mathrm{keV}$. The proton cyclotron features (PCFs) are conspicuous (equivalent width of up to many hundreds $\mathrm{eV}$ ) and relatively broad $(\Delta E / E \sim 0.05-0.2)$. The detection of the PCFs is well within the capabilities of present X-ray spectrometers, like the HETGS and METGS on board Chandra. Their observation might provide decisive evidence in favor of the existence of magnetars.
\end{abstract}

Subject headings: radiative transfer - stars: neutron - X-ray: stars

\section{INTRODUCTION}

Over the last few years increasing observational evidence has gathered in favor of the existence of ultramagnetized neutron stars (NSs) with surface field $B \gtrsim 10^{14} \mathrm{G}$. The existence of these magnetars was first proposed nearly 10 years ago by Thompson \& Duncan (1993) who showed that, during the core collapse, convective motions can strongly amplify the seed magnetic field.

Magnetars are unique among compact stars, in that their luminosity should be provided by the dissipation of magnetic energy. Like conventional radiopulsars, however, they will also lose rotational energy according to the magnetic dipole formula. Owing to their high magnetic field, magnetars are expected to spin down at a high rate, $\dot{P} \approx 10^{-11}\left(B / 10^{14} \mathrm{G}\right)^{2} / P$ $\mathrm{ss}^{-1}$. It was the detection of pulsations with a secular spin-down in this range in two soft $\gamma$-repeaters (SGRs; Kouveliotou et al. 1998; 1999) that for the first time suggested the association of these sources with ultra-magnetized NSs. Besides their bursting activity, SGRs show also persistent X-ray emission with luminosities $L \approx 10^{34}-10^{36} \mathrm{erg} \mathrm{s}^{-1}$. X-ray spectra are usually power-law like, but in one case (SGR 1900+14) clear evidence was found for a ther- mal spectral component at $k T \sim 0.5 \mathrm{keV}$ (Woods et al. 1999). This is believed to originate from the NS surface radiating away the dissipated magnetic energy (see e.g. Thompson 2000 for a recent review).

Magnetars have been also invoked to explain the characteristics of another enigmatic class of Galactic sources, the so-called anomalous X-ray pulsars (AXPs). These pulsars share properties that are much at variance with those of canonical X-ray pulsators (Mereghetti \& Stella 1995). Among these are: i) a narrow interval of pulse periods (6-12 s); ii) very soft X-ray spectra, often with a blackbodylike component at $k T \sim 0.4-0.6 \mathrm{keV}$; iii) relatively low X-ray luminosities ( $\left.10^{35} \mathrm{erg} \mathrm{s}^{-1}\right)$; iv) spindown trends at relatively large and stable rates $(\sim$ $\left.0.6-30 \times 10^{-12} \mathrm{~s} \mathrm{~s}^{-1}\right)$; v) absence of massive companion stars and vi) association with supernova remnants (see e.g. Mereghetti 2000 for a review).

Though it is now widely recognized that AXPs are rotating NSs, two different mechanisms for powering their X-ray emission have been discussed: accretion (from a very low mass companion, fall back or a fossil disk, Van Paradijs, Taam, \& Van den Heuvel 1995; Chatterjee, Hernquist, \& Narayan 2000) and magnetic energy dissipation in ultra-magnetized, isolated

\footnotetext{
${ }^{1}$ Mullard Space Science Laboratory, University College London, Holmbury St. Mary, Dorking, Surrey, RH5 6NT, UK; e-mail: sz@mssl.ucl.ac.uk

${ }^{2}$ Dipartimento di Fisica, Università di Padova, Via Marzolo 8, I-35131 Padova, Italy; e-mail: turolla@pd.infn.it

${ }^{3}$ Osservatorio Astronomico di Roma, via dell'Osservatorio 2, I-00040 Monte Porzio Catone, Roma, Italy; e-mail: stella@coma.mporzio.astro.it

${ }^{4}$ Dipartimento di Scienze, Università dell'Insubria, Via Valleggio 11, Como, Italy; e-mail: treves@mib.infn.it 
NSs. If AXPs are young, hot NSs giving off thermal radiation, magnetic field decay should provide crustal heating for a long enough time. Possible decay scenarios have been analyzed by Colpi, Geppert \& Page $(2000)$ and led to the conclusion that AXPs are $\approx 10^{4}$ yr old with a present $B$-field of $\approx 10^{14} \mathrm{G}$. The latter figure is in the range of that inferred from SGRs spindown rates. The many similarities between AXPs and SGRs strengthen the idea that the two classes of sources are powered by the same mechanism, i.e. magnetic field dissipation (e.g. Thompson 2000).

The transfer of radiation in strongly magnetized atmospheres has been widely investigated in connection with X-ray emission from cooling and accreting neutron stars (see e.g. Shibanov et al. 1992; Rajagopal, \& Romani 1996; Pavlov et al. 1996; Zane, Turolla \& Treves 2000, herafter ZTT). Up to now, however, little attention was given to emission from ultra-magnetized neutron stars with surface fields $z 10^{13} \mathrm{G}$. Detailed transfer calculations to assess the emergent spectrum from atmospheres around ultramagnetized NSs are therefore needed. Model fits to the X-ray spectra of AXPs and SGRs indicate the presence of a power-law, high-energy component. In addition to this, thermal emission has been detected in all AXPs but one, and in SGR 1900+14 (see Mereghetti 2000 and references therein).

In this paper we present numerical models of thermal emission from NSs for a range of luminosity, $L \sim 10^{34}-10^{36} \mathrm{ergs}^{-1}$, and magnetic field, $B \sim$ $10^{14}-10^{15} \mathrm{G}$, which are thought typical of SGRs and AXPs. Emergent spectra, computed solving the full (angle and energy dependent) transfer equations, are nearly Planckian, have negligible hardening, and exhibit a strong absorption feature at the proton cyclotron resonance, $E_{c, p} \simeq 0.63 y_{G}\left(B / 10^{14} \mathrm{G}\right) \mathrm{keV}$, where $y_{G}=\sqrt{1-2 G M / c^{2} R}$ is the gravitational redshift factor ( $M$ and $R$ are the star mass and radius, respectively). The predicted equivalent width of the proton cyclotron features (PCFs) is in the range 0.03$1 \mathrm{keV}$ and the relative width $F W Z I / E_{c, p} \approx 0.7$ (here $F W Z I$ is the full width at zero intensity). The detection of PCFs is definitely within the range of Chandra spectrometers. Their actual observation in the X-ray spectra of SGRs and/or AXPs would provide a direct measurement of the surface magnetic field and a remarkable confirmation of the magnetar model.

\section{MODEL ATMOSPHERES}

In this paper we follow closely the approach presented in ZTT, to which we refer for details. In particular we consider a magnetized, non-degenerate, pure hydrogen plasma in thermal equilibrium at temperature $T$ in which the dominant radiative processes are free-free emission/absorption and Thomson scatter- ing; non-conservative scattering has negligible effect on the transfer of radiation but becomes important in the energy balance of the outer layers (see below). We approximate the atmosphere as a plane-parallel slab with the magnetic field $\mathbf{B}$ parallel to the normal $\mathbf{n}$ (the $z$-axis). This is reasonable for magnetized accretors, but it becomes questionable in treating emission from the entire star surface, as in the case of cooling neutron stars. The effects induced on the emerging spectrum by including surface elements away from the magnetic poles are therefore discussed in section 3. Although we are mainly interested in thermal emission from magnetars, ultra-magnetized NSs might also accrete the interstellar medium, as recently suggested by Rutledge (2001). In the light of this, and for the sake of completeness, we consider here both the case of accreting and cooling atmospheres.

For photon frequencies in the range $\omega_{p}<\omega<\omega_{c, e}$, where $\omega_{p}$ and $\omega_{c, e}=e B / m_{e} c$ are the plasma and the electron cyclotron frequency respectively, the semitransverse approximation can be used and the transfer of radiation through the magneto-active medium is described in terms of the two normal modes (ZTT and references therein). Atmospheric models are constructed by solving the transfer equations for the normal modes coupled to the hydrostatic equilibrium and the energy equation. Since the luminosity $L$ is well below the Eddington limit, the hydrostatic balance gives immediately the density as a function of the scattering depth $\tau=\int_{\infty}^{z} \kappa_{e s} \rho d z$

$$
\rho=\frac{G M m_{p}}{2 y_{G}^{2} R^{2} \kappa_{e s}} \frac{\tau}{k T(\tau)}
$$

(here $\kappa_{e s}=0.4 \mathrm{~cm}^{2} \mathrm{~g}^{-1}$ is the electron scattering opacity for an unmagnetized medium; see ZTT). The energy balance simply states that the net radiative cooling must equate the energy $W_{H}$ released in the atmosphere by non-radiative processes, like the bombardment of impinging ions; for cooling atmospheres $W_{H}=0$. Including Compton heating/cooling the energy equation becomes

$$
k_{P} \frac{a T^{4}}{2}-\sum_{i=1}^{2} k_{a m}^{(i)} U^{(i)}+(\Gamma-\Lambda)_{C}=\frac{W_{H}}{c \kappa_{e s}}
$$

where $U^{(i)}$ is the radiation energy density of mode $i$, $k_{P}, k_{a m}^{(i)}$ are defined in strict analogy with the Planck and absorption mean opacities in the unmagnetized case and $(\Gamma-\Lambda)_{C}$ is the Compton heating/cooling rate in a magnetized plasma (see again ZTT). 


\section{RESULTS}

Following the method outlined in the previous section, several model atmospheres have been computed for different values of the magnetic field strength and luminosity. As expected (see ZTT; Treves et al. 2000), cooling and accreting atmospheres turn out to produce essentially the same spectrum at Xray energies, so we do not make further distinction between them. It should be kept in mind, however, that a cooling model characterized by a luminosity $L$ is equivalent to an accreting one with luminosity $f_{A} L<L$, where $f_{A}$ is the fraction of the emitting area. All models have been calculated for $M=1 M_{\odot}$, $R=9 \mathrm{~km}$ and are labeled by the value of the luminosity measured by an observer at infinity, assuming emission from the entire surface (i.e. $f_{A}=1$ ). We have explored the ranges $10^{13} \mathrm{G} \leq B \leq 10^{15} \mathrm{G}$ and $10^{34} \mathrm{erg} \mathrm{s}^{-1} \lesssim L \lesssim 10^{36} \mathrm{erg} \mathrm{s}^{-1}$ which are believed typical of magnetars. The main parameters of the computed models are summarized in table 1 and emerging spectra are shown in figure 1. In all cases, with the exception of model A4, the spectra are nearly Planckian in shape and show a small hardening with respect to the blackbody at the star effective temperature $T_{e f f}$. As it was found in previous investigations (e.g. Shibanov et al. 1992; ZTT), the spectral hardening decreases with increasing field strength and almost vanishes for $B \gtrsim 10^{14} \mathrm{G}$. The most prominent spectral signature is the absorption feature at the proton cyclotron resonance $E_{c, p} \simeq$ $0.63 y_{G}\left(B / 10^{14} \mathrm{G}\right) \mathrm{keV}$ which falls in the soft-medium $\mathrm{X}$-rays for $B \sim 10^{14}-10^{15} \mathrm{G}$; the electron cyclotron line is at $\simeq 1.2\left(B / 10^{14} \mathrm{G}\right) \mathrm{MeV}$. The line equivalent width, $E W$, and the full width at zero intensity relative to the line center, $\Delta E / E \equiv F W Z I / E$, are reported in table 11. Two main effects contribute to the line profile: the intrinsic resonance in the magnetic absorption coefficients (this essentially produces Fraunhöfer lines) and mode crossing at the mode collapse points. The latter is amplified when collapse points induced by vacuum effects fall near the resonance and in the photospheric region (see also Shibanov et al. 1992). The large modification in the continuum in model A4, for instance, is partially due to mode-crossing which, for these values of the parameters, occurs close to the Wien peak.

As discussed earlier, the assumption of a constant magnetic field breaks down when emission comes from the entire star surface. Even for a simple dipolar field, $\mathbf{B}$ changes in both magnitude and direction along the surface and this will produce a broadening of the PCF. Moreover, large $B$-fields introduce a meridional temperature variation that makes the emergent spectrum dependent of the viewing angle. A thorough modeling would require a genuine two- dimensional transfer calculation, which is still beyond the capabilities of present numerical codes. However, an estimate of the expected broadening can be obtained with a simple, approximated computation. To do this, we assume a relativistic magnetic dipole in the Schwarzschild space-time, aligned with the rotational axis (see e.g. Pavlov \& Zavlin 2000)

$$
B=\frac{B_{p}}{2}\left[\left(4-f^{2}\right) \cos ^{2} \chi+f^{2}\right]^{1 / 2}
$$

where $B_{p}$ is the field strength at the magnetic pole $(\chi=0)$ and $f \simeq 1.1$ accounts for gravitational effects. The surface temperature profile is taken from Possenti, Mereghetti \& Colpi (1996)

$$
T=T_{\text {eff }}\left\{\frac{K+(4-K) \cos ^{2} \chi}{[1-0.47(1-K)]\left(1+3 \cos ^{2} \chi\right)}\right\}^{1 / 4}
$$

where $K$ is the ratio of the coefficients of thermal conductivity orthogonal and parallel to the field. The exact value of $K$ is not very important: for $B \gtrsim 10^{13}$ $\mathrm{G}$ it is $K \lesssim 10^{-4}$, and beyond this value the temperature profile depends weakly on $K$. The flux emitted by the surface element at co-latitude $\chi$ is evaluated assuming thermal diffusion for both modes

$$
d F_{\nu}=2 \pi \sum_{i=1}^{2} \frac{D^{(i)}}{2} \frac{d B_{\nu}[T(\chi)]}{d \tau} \sin \chi d \chi
$$

where $B_{\nu}$ is the blackbody function. The diffusion coefficients $D^{(i)}$ are obtained by angle-averaging the reciprocal of the total opacity over all photon directions (see Shibanov et al. 1992). They depend on $\mu_{B}=\mathbf{n} \cdot \mathbf{B} / B$; here $\mathbf{n}=\mathbf{r} / r$ is the "local" normal to the surface. The total flux is then computed by integrating equation (5) over the entire star surface, with the aid of expression

$$
\mu_{B}^{2}=\frac{1}{4-f^{2}}\left[4-\frac{4 f^{2}}{\left(4-f^{2}\right) \cos ^{2} \chi+f^{2}}\right]
$$

which relates $\mu_{B}$ to $\chi$ (see again Pavlov \& Zavlin 2000).

Line parameters derived from the previous calculation are reported in table 2 for different values of the luminosity and of the polar $B$-field; diffusion-limit spectra, as seen at the top of the NS atmosphere, are shown in figures 24. For $B_{p}=10^{15} \mathrm{G}$ the PCF falls in the Wien tail and it is spread over a few keVs. To allow a direct comparison with the case of a magnetic field parallel to the surface normal, we have repeated the same calculation for a planeparallel slab with constant $B=B_{p}$, neglecting surface temperature variations. We stress that in both cases no mode crossing effects have been accounted 
TABLE 1

Model Parameters

\begin{tabular}{cccccc}
\hline \hline Model & $\begin{array}{c}B \\
10^{13} \mathrm{G}\end{array}$ & $\begin{array}{c}L \\
10^{34} \mathrm{erg} \mathrm{s}^{-1}\end{array}$ & $\begin{array}{c}E_{c, p}^{a} \\
\mathrm{keV}\end{array}$ & $\begin{array}{c}E W \\
\mathrm{keV}\end{array}$ & $\Delta E / E$ \\
\hline $\mathrm{A} 1$ & 1 & 2 & 0.06 & 0.01 & 0.67 \\
$\mathrm{~A} 2$ & 1 & 70 & 0.06 & 0.01 & 0.47 \\
$\mathrm{~A} 3$ & 5 & 9 & 0.32 & 0.05 & 0.31 \\
$\mathrm{~A} 4$ & 10 & 3 & 0.63 & - & - \\
$\mathrm{A} 5$ & 50 & 170 & 3.15 & 0.10 & 0.10 \\
$\mathrm{~A} 6$ & 100 & 180 & 6.3 & 0.11 & 0.06 \\
& & & & & \\
\hline
\end{tabular}

Note. - The line energy is not corrected for the gravitational red-shift; the corresponding value at Earth is lower by a factor $y_{G} \sim 0.8$.

for, and, to be consistent with the diffusion approximation, spectra were computed at optical depth $\gtrsim 1$. Clearly these approximated results may be taken only as indicative of the true shape of the PCF in emergent spectra; it is however worth noticing that the line equivalent widths derived from our simple analysis for $\mathbf{B} \| \mathbf{n}$ are in rough agreement with those of the complete transfer calculation for the same values of $B$ and $L$. As expected, the PCF turns out to be systematically broader when emission comes from the entire star surface, typically by $10-20 \%$. Also, the change of the field strength with the latitude produces a shift of the line centroid toward lower energies by $\sim 20-30 \%$. Both these effects are quite independent of the values of $B_{p}$ and $L$ (see table 2). Complex magnetic field configurations with contributions from higher multipoles, such as those envisaged in magnetar models (Thompson 2000) or in the modelling of isolated neutron stars (e.g. Geminga, Page et al. 1995), perhaps represent a situation more realistic than the dipolar field we have considered. Due to the steep effective temperature gradients expected in this case, the real properties of PCFs should be intermediate between those discussed above.

\section{DISCUSSION}

In this paper we have modeled thermal emission from ultra-magnetized NSs, showing that the emergent continuum is almost indistinguishable from a blackbody at $T_{\text {eff }}$. We point out the existence of a strong absorption feature at the proton cyclotron energy; the line equivalent width is between $30 \mathrm{eV}$ and
$1 \mathrm{keV}$ and the line center is located at $\sim 0.5-5 \mathrm{keV}$ for field strengths $\sim 10^{14}-10^{15} \mathrm{G}$, which are currently believed to be typical of SGRs and AXPs. The radiative transfer calculations presented here corroborate the original suggestion by Thompson (2000) that an emission/absorption feature at the proton cyclotron resonance should be present in the thermal spectrum of magnetars.

The natural width and the line-splitting of electron cyclotron lines were thoroughly investigated by Pavlov et al. (1991) by means of a rigorous QED treatment. No similar calculation is available for protons, but, in any realistic situation, $B \ll$ $B_{\text {crit }, p}=m_{p}^{2} c^{3} / \hbar e \sim 1.5 \times 10^{20} \mathrm{G}$, so protons are never relativistic. In this case, the natural width of the line associated with the transition between the first and the ground Landau levels is $\Gamma_{1}^{ \pm} \sim$ $(4 / 3) \alpha m_{p} c^{2}\left(B / B_{\text {crit }, p}\right)^{2}$ (Pavlov, private communication), where $\alpha$ is the fine structure constant and the plus/minus sign refers to spin-up $\leftrightarrow$ spin-up, spindown $\leftrightarrow$ spin-down transitions respectively. In addition to the first order cyclotron resonance Thompson (2000) also suggested the possibility to detect the spin-flip line, despite the fact that no detailed calculation has been presented. In fact, contrary to electrons, which have a small anomalous magnetic moment $\Delta \mu / \mu_{0} \approx \alpha$ (here $\mu_{0}$ is the Böhr magneton), $\Delta \mu / \mu_{N} \simeq 2.79$ for protons, $\mu_{N}=\left(m_{p} / m_{e}\right) \mu_{0}$ being the nuclear magneton. This implies that the proton spin-flip transition produces a line at $2.79 E_{c, p}$ which is always well-separated from the fundamental resonance at $E_{c, p}$. Here we only note that this 
TABLE 2

Line BRoAdEning

\begin{tabular}{ccccccc}
\hline \hline $\begin{array}{c}B_{p} \\
10^{13} \mathrm{G}\end{array}$ & $\begin{array}{c}L \\
10^{34} \mathrm{erg} \mathrm{s}^{-1}\end{array}$ & $\begin{array}{c}E W_{D} \\
\mathrm{keV}\end{array}$ & $E W_{D} / E W_{\|}$ & $\begin{array}{c}E_{c, D} \\
\mathrm{keV}\end{array}$ & $E_{c, D} / E_{c, \|}$ & $\Delta E_{D} / E_{c, D}$ \\
\hline 1 & 0.1 & 0.035 & 1.17 & 0.046 & 0.73 & 0.84 \\
1 & 1 & 0.035 & 1.20 & 0.046 & 0.73 & 0.84 \\
1 & 10 & 0.035 & 1.22 & 0.047 & 0.75 & 0.80 \\
10 & 0.1 & 0.35 & 1.14 & 0.49 & 0.78 & 0.75 \\
10 & 1 & 0.35 & 1.12 & 0.47 & 0.75 & 0.86 \\
10 & 10 & 0.34 & 1.08 & 0.46 & 0.73 & 0.73 \\
50 & 1 & 1.74 & 1.06 & 2.57 & 0.81 & 0.53 \\
50 & 10 & 1.72 & 1.06 & 2.72 & 0.86 & 0.63 \\
50 & 100 & 1.75 & 1.11 & 2.49 & 0.79 & 0.79 \\
& & & & & & \\
\hline
\end{tabular}

Note. $-D=$ dipolar field; $\|=$ constant $B$ field. $E_{c}$ is the line centroid and $\Delta E_{D}$ is the FWZI in the case of dipolar field; energies have not been corrected for the gravitational red-shift.

second line should be much weaker and is probably beyond the capabilities of the present spectrometers, because the rates for the spin-flip transitions in the non-relativistic regime should contain an additional factor $\approx E_{c, p} / m_{p} c^{2} \ll 1$ with respect to transitions with conserved spin projection (Pavlov, private comunication; see also Melrose \& Zheleznyakov 1981 for the case of electrons). On the other hand, the fact that the lowest Landau level is always non-degenerate for protons might prove decisive in discriminating between proton and electron absorption features in high-resolution spectral observations. In fact, if absorption features observed at $0.06-6 \mathrm{keV}$ are interpreted as electron cyclotron resonances, the inferred magnetic fields should be as low as $10^{9}-10^{11} \mathrm{G}$. For values in this range, the interaction with the radiation field should remove the degeneracy of the lowest Landau level, giving rise to a doublet. In the case of electrons, even if the two lines are not individually resolved (the required resolving power should be $\left.\sim E_{c, e} / \Delta E \sim(0.16 \alpha)^{-1} \sim 860\right)$ observations with a narrow band Bragg polarimeter should show a sharp jump when scanning the feature from the blue toward the red wing (see Pavlov et al. 1991 for details).

Finally, we would like to stress that present results have been derived under a set of simplifying assumptions, on both the atmospheric properties and the line profiles. Atmospheres around young NSs are likely to be rich in metals. Unfortunately the absorption coefficients for metals in a strong magnetic field are poorly known and our assumption of a pure $\mathrm{H}$ composition, which is certainly inadequate, appears at present the only viable option. Metal lines may well be present in magnetars X-ray spectrum. If originating in an accreting gas or a corona they are expected to be mainly in emission and narrower, unless the circumstellar material where the line originates has a velocity large enough to produce a (Doppler-) broadening comparable to that of the PCF. This is not obvious if the NS atmosphere itself is rich in heavy elements since energies and shapes of atomic transitions are strongly dependent on the local conditions (see Rajagopal, \& Romani 1996; Zavlin et al. 1996). Strongly magnetized Fe spectra presented by Rajagopal, Romani, \& Miller (1997) show significant absorption features in the X-rays. A confusion between metal lines and PCFs is therefore possible and this issue should be carefully addressed when observed spectra are analyzed. Moreover, for a different chemical composition, lower energy cyclotron lines at the various ion resonances should also be present, since $E_{c, i}=(Z / A) E_{c, p}$. A firm identification of an observed feature in terms of a PCF may follow from the detection of higher harmonics (already observed in some pulsars for the electron cyclotron resonance), despite they are expected to be weaker (but see Cosumano et al. 1998 for a counterexample). In the normal mode approximation, higher-order harmonics 
of the electron cyclotron resonance can be accounted for in the Gaunt factors (see Pavlov \& Panov 1976; these expressions have been used here and in ZTT), but we are unaware of a similar treatment of the effective collision frequencies for the higher harmonics of the proton resonance.

Partial $\mathrm{H}$ ionization should also be taken into account. Although no detailed investigation on the ionization properties of hydrogen in ultra-strong fields have been carried out, the results of Potekhin, Chabrier \& Shibanov (1999) for $B \lesssim 10^{13} \mathrm{G}$ clearly indicate that the neutral fraction increases with the field strength. For values of density and temperature typical of the inner layers of our atmospheric models, the neutral fraction is $\approx 0.01$ at $B=10^{13} \mathrm{G}$ and it is bounded to increase at larger $B$. This will reduce the number of free protons, thus making resonant absorption less effective.

Line-broadening mechanisms have not been accounted for in our present investigation, apart from the smearing of the line introduced by the dipolar variation of the $B$-field (see $\xi_{3}$ ). The more important broadening could be due to the thermal Doppler effect, which produces a width $\Delta E_{t h} \approx$ $E_{c, p}\left(k T / m_{p} c^{2}\right)^{1 / 2} \approx 10^{-4} E_{c, p}$ under typical conditions. As expected, Doppler-broadening is less important by far for protons with respect to electrons and $\Delta E_{t h} \ll \Delta E$ in our models (see table 2).
Up to now no positive detection of any spectral feature, both in emission and in absorption, have been reported in SGRs and AXPs (see Mereghetti 2000 and references therein). The only possible exception is the anomalous X-ray pulsar $1 \mathrm{E} 2259+586$, for which the detection of a cyclotron feature around 5 $\mathrm{keV}$ has been claimed (Iwasawa, Koyama \& Halpern 1992) but never confirmed by subsequent observations (e.g. Corbett et al. 1995). The discrepancy between the spin-down age and the age of the SNR in this source hints toward a higher value of the field in the past, but the large line energy would imply a present field $\approx 10^{15} \mathrm{G}$, which seems difficult to reconcile with the pulsar evolutionary track (Colpi, Geppert \& Page 2000). However, present data do not exclude the possibility that in SGRs and AXPs the magnetic field is a few $10^{14} \mathrm{G}$. If this is the case, a cyclotron feature around $\sim 1 \mathrm{keV}$ may well have escaped detection because these sources have never been observed with sufficient spectral resolution at low energies.

We are grateful to G.G. Pavlov for several enlightening comments on the radiative width of the proton cyclotron line and the relative importance of the proton spin-flip transition. Work partially supported by Italian MURST under grant COFIN-98-02154100.

\section{REFERENCES}

Chatterjee, P., Hernquist, L., \& Narayan, R. 2000, ApJ, 534, 373

Colpi, M., Geppert, U., \& Page, D. 2000, ApJ, 529, L29

Corbett, R.H.D. et al. 1995, ApJ, 443, 786

Cosumano, G. et al. 1998, A\&A, 338, L79

Iwasawa, K., Koyama, K., \& Halpern, J.P. 1992, PASJ, 44, 9

Kouveliotou, C. et al. 1998, Nature, 393, 235

Kouveliotou, C. et al. 1999, ApJ, 510, L115

Melrose, D.B., \& Zheleznyakov, V.V. 1981, A\&A, 95, 86

Mereghetti, S. 2000, in The Neutron Star-Black Hole Connection, eds. V. Connaughton, C. Kowveliotow. I. Van

Paradijs \& J. Ventura (NATO-ASI), astro-ph/9911252

Mereghetti, S., \& Stella, L. 1995, ApJ, 442, L17

Page, D., Shibanov, Yu.A., \& Zavlin, V.E. 1995, ApJ, 451, L21

Pavlov, G.G., Bezchastnov, V.G., Mészáros, P., \& Alexander, S.G. 1991, ApJ, 380, 541

Pavlov, G.G., \& Panov, A.N. 1976, Sov. Phys. JETP, 44, 300

Pavlov, G.G., Zavlin, V.E., Trümper, J., \& Neuhaeuser, R. 1996, ApJ, 472, L33

Pavlov, G.G., \& Zavlin, V.E. 2000, ApJ, 529, 1011

Possenti, A., Mereghetti, S., \& Colpi, M. 1996, A\&A, 313, 565
Potekhin, A.Y., Chabrier, G., \& Shibanov, Yu.A. 1999, Phys. Rev. E, 60, 2193

Rajagopal, M., \& Romani, R.W. 1996, ApJ, 461, 327

Rajagopal, M., Romani, R.W., \& Miller, M.G. 1997, ApJ, 479, 347

Shibanov, Yu.A., Zavlin, V.E., Pavlov, G.G., \& Ventura, J. 1992, A\&A, 266, 313

Rutledge, R.E. 2001, ApJ, in the press, astro-ph/0101550

Thompson, C.. \& Duncan, R.C. 1993, ApJ, 408, 194

Thompson, C. 2000, in The Neutron Star-Black Hole Connection, eds. V. Connaughton, C. Kouveliotou, J. Van Paradijs \& J. Ventura (NATO-ASI), astro-ph/0010016

Treves, A., Turolla, R., Zane. S., \& Colpi, M. 2000, PASP, 112, 297

Van Paradijs, J., Taam, R.E., \& van den Heuvel, E.P.J. 1995, A\&A, 299, L41

Zane, S., Turolla, R., \& Treves, A. 2000, ApJ, 537, 387 (ZTT)

Zavlin, V.B., Pavlov, G.G., \& Shibanov, Yu.A. 1996, A\&A, 315,

Woods, P. et al. 1999, ApJ, 518, L103 

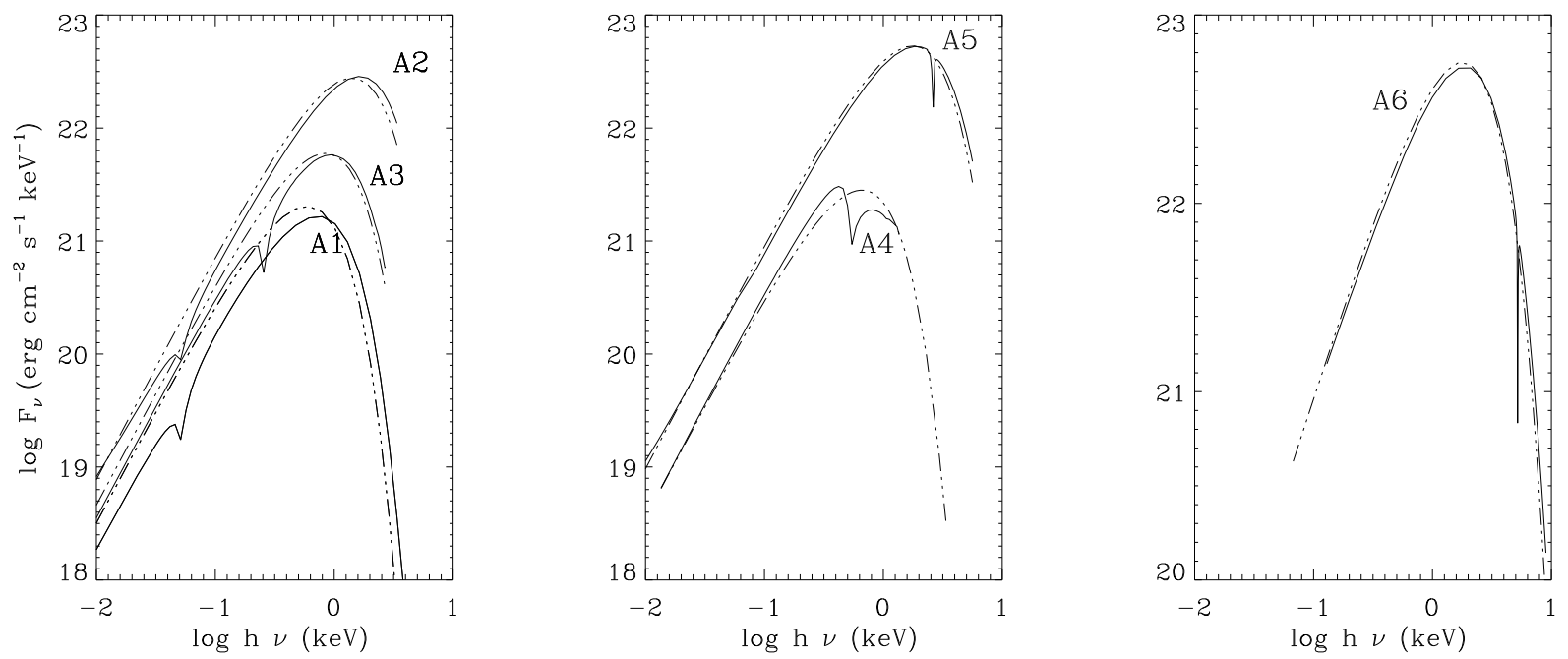

FIG. 1.- Emergent spectra (solid lines) for the models presented in table 1, together with the blackbody at the NS effective temperature (dash-dotted lines). Energies have been red-shifted at Earth while the monochromatic flux (on the vertical axis) is evaluated at the star surface.
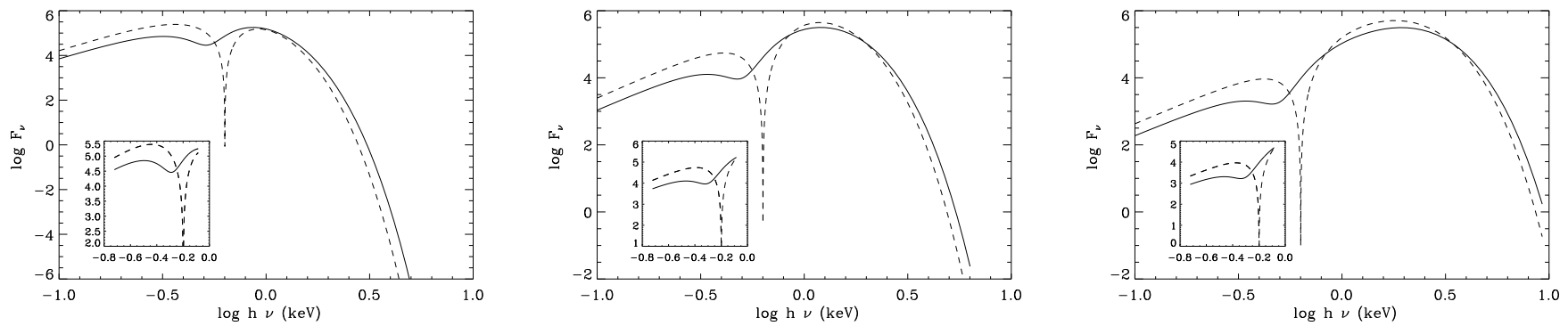

Fig. 2.- Broadening of the proton cyclotron line for $B=10^{14} \mathrm{G}$ and different luminosities. From left to right: $L=$ $10^{33}, 10^{34}, 10^{35} \mathrm{erg} \mathrm{s}^{-1}$; solid lines correspond to a dipolar field, and dash-dotted lines to $B$ normal to the surface. Flux is in arbitrary units and energies have not been red-shifted at Earth.
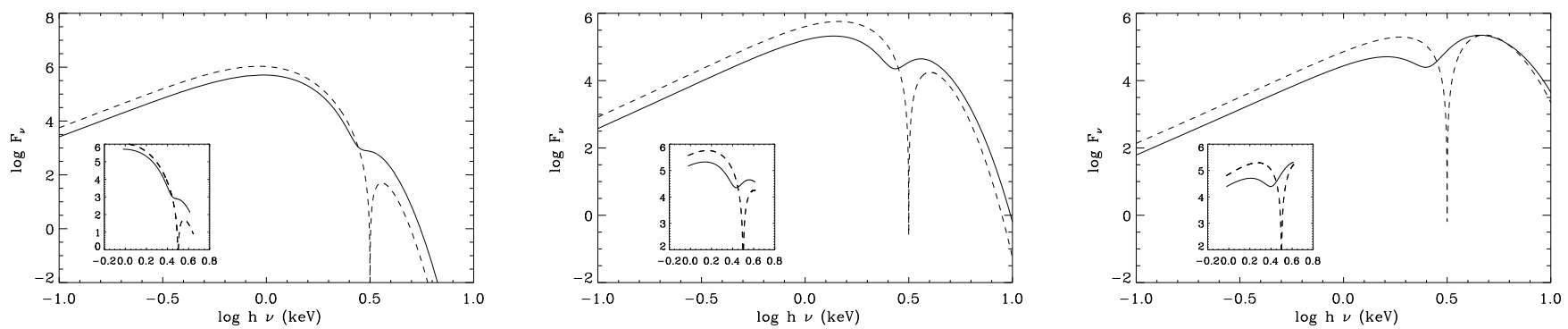

FIG. 3.- Same as in figure 2 2 for $B=5 \times 10^{14} \mathrm{G}$ and $L=10^{34}, 10^{35}, 10^{36} \mathrm{erg} \mathrm{s}^{-1}$.
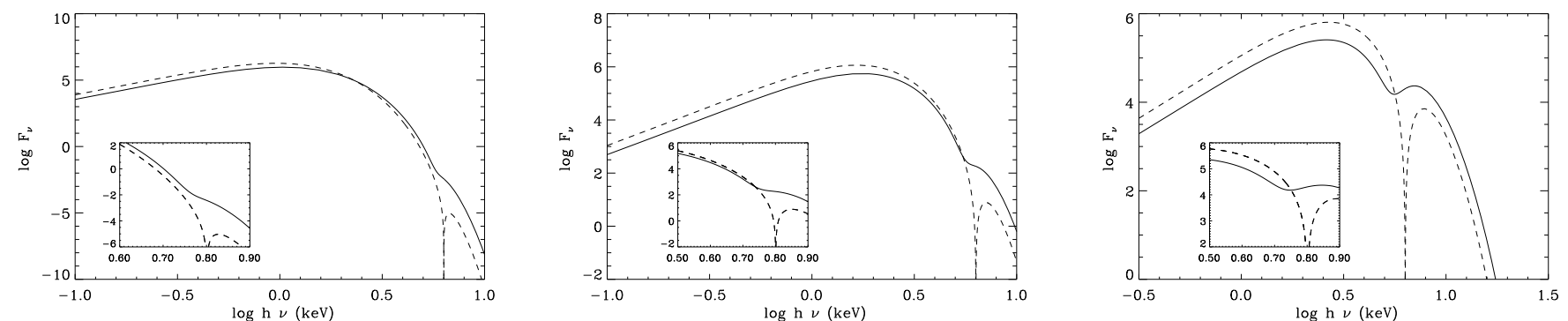

Fig. 4.- Same as in figure 3 for $B=10^{15} \mathrm{G}$. 